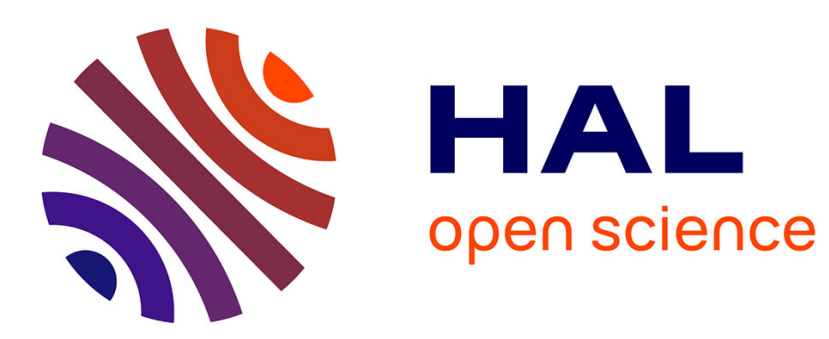

\title{
A Fuzzy Approach for Background Subtraction
}

Fida El Baf, Thierry Bouwmans, Bertrand Vachon

\section{To cite this version:}

Fida El Baf, Thierry Bouwmans, Bertrand Vachon. A Fuzzy Approach for Background Subtraction. ICIP 2008, Oct 2008, San Diego, United States. pp.2648-2651. hal-00340190

\section{HAL Id: hal-00340190 https://hal.science/hal-00340190}

Submitted on 20 Nov 2008

HAL is a multi-disciplinary open access archive for the deposit and dissemination of scientific research documents, whether they are published or not. The documents may come from teaching and research institutions in France or abroad, or from public or private research centers.
L'archive ouverte pluridisciplinaire HAL, est destinée au dépôt et à la diffusion de documents scientifiques de niveau recherche, publiés ou non, émanant des établissements d'enseignement et de recherche français ou étrangers, des laboratoires publics ou privés. 


\title{
A FUZZY APPROACH FOR BACKGROUND SUBTRACTION
}

\author{
Fida El Baf, Thierry Bouwmans, Bertrand Vachon \\ Department of Mathematics, Image and Applications \\ University of La Rochelle, France \\ felbaf@univ-lr.fr
}

\begin{abstract}
Background Subtraction is a widely used approach to detect moving objects from static cameras. Many different methods have been proposed over the recent years and can be classified following different mathematical model: determinist model, statistical model or filter model. The presence of critical situations i.e. noise, illumination changes and structural background changes introduce two main problems: The first one is the uncertainty in the classification of the pixel in foreground and background. The second one is the imprecision in the localization of the moving object. In this context, we propose a fuzzy approach for background subtraction. For this, we use the Choquet integral in the foreground detection and propose fuzzy adaptive background maintenance. Results show the pertinence of our approach.
\end{abstract}

Index Terms - Background Subtraction, Background Maintenance, Foreground Detection, Choquet Integral

\section{INTRODUCTION}

Background subtraction is commonly used in the field of video surveillance [3], optical motion capture [4], and multimedia application [2] where it needs in the first step to detect the moving objects in the scene. The basic idea is to classified pixel as background or foreground by thresholding the difference between the background image $B_{t}(x, y, t)$ and the current image $I_{t+1}(x, y, t)$. Due of the presence of critical situations, false positive or negative detection appear corresponding to false classification of pixels. To decrease these effects, the different background subtraction methods have been developed and can be classified following the model used in the background representation step:

- Basic Background Modeling: the average [5] or the median [6] or the histogram analysis over time [7].

- Statistical Background Modeling: the single Gaussian [8] or the Mixture of Gaussians [9] or the Kernel Density Estimation [10].

- Background Estimation: Wiener filter [11], Kalman filter [12] or Tchebychev filter [13].
All these methods have the following steps:Background Modeling, Background Initialization, Background Maintenance and Foreground Detection. Developing a background subtraction method, researchers must design each step and choose the features size (pixel, a block or a cluster) and type (color, edge, stereo, motion and texture) in relation to the critical situations they want to handle. In this article, we focus on the foreground detection and the background maintenance using color features. In Section 2, we propose to use the Choquet integral in the foreground detection. In the section 3, we enforce spatial consistency. In the section 4 , we propose an adaptive scheme for the background maintenance. Finally in the section 5, we present results on video datasets.

\section{FOREGROUND DETECTION USING FUZZY INTEGRAL}

In the litterature, many features are used for the detection of moving objects. In the case of color features, some authors make the foreground detection in each dimension independently and then aggregate the corresponding foreground mask using the binary operator (OR). The disadvantage is that a false positive in one dimension generate false positive in the final result. We propose thus to use a fuzzy operator i.e. the Choquet integral to aggregate the results obtained in each dimension to avoid crisp decision. In the following subsections, we first present the color similarity measure. Then, we summarize briefly concepts around fuzzy integrals and finally apply the Choquet integral to aggregate the similarity measure computed in different dimensions.

\subsection{Color Similarity Measure}

We describe here the similarity measure in a general way, i.e the color features may be any color space with three components noted $C_{1}, C_{2}$ and $C_{3}$. Then, the color similarity measure $S_{k}^{C}(x, y)$ at the pixel $(x, y)$ is computed as in [1]:

$$
S_{k}^{C}(x, y)=\left\{\begin{array}{lll}
\frac{I_{k}^{C}(x, y)}{I_{k}^{B}(x, y)} & \text { if } & I_{k}^{C}(x, y)<I_{k}^{B}(x, y) \\
1 & \text { if } & I_{k}^{C}(x, y)=I_{k}^{B}(x, y) \\
\frac{I_{k}^{B}(x, y)}{I_{k}^{C}(x, y)} & \text { if } & I_{k}^{C}(x, y)>I_{k}^{B}(x, y)
\end{array}\right.
$$


where $k \in\{1,2,3\}$ is one of the three color features, $B$ and $C$ represent respectively the background and the current frame at time $t$. $B$ can be obtained using any of the background modelling method. Note that $S_{k}^{C}(x, y)$ is between 0 and 1. Futhermore, $S_{k}^{C}(x, y)$ is close to one if $I_{k}^{C}(x, y)$ and $I_{k}^{B}(x, y)$ are very similar.

\subsection{Fuzzy Integral}

Let $\mu$ be a fuzzy measure on a finite set $X$ of criteria and $h: X \rightarrow[0,1]$ be a fuzzy subset of $X$.

Definition 1 The Choquet integral of $h$ with respect to $\mu$ is defined by:

$$
C_{\mu}=\sum_{i=0}^{n} h\left(x_{\sigma(i)}\right)\left(\mu\left(A_{\sigma(i)}\right)-\mu\left(A_{\sigma(i+1)}\right)\right)
$$

where $\sigma$ is a permutation of the indices such that

$h_{\sigma(1)} \leq \ldots \leq h_{\sigma(n)}$ and $A_{\sigma(i)}=\{\sigma(1), \ldots, \sigma(n)\}$

In a recent study [17], we have proved that the use of the Choquet integral for fusing features in foreground detection problem has provide improved over the Sugeno integral. We have proved in a recent study [17] that Choquet integral is more suitable than Sugeno one to aggregate these features. More details can be found in ([14], [15]). So we opted for the Choquet integral to aggregate different dimensions for a chosen color space as explained in the following subsection.

\subsection{Aggregation of color features by Choquet Integral}

Foreground objects can be made by the fusion of different features with a Choquet integral. For each pixel, similarity measures are computed in different dimension from the background and the current frame as explained in section 2.1. We defined the set of criteria $X \in\left\{x_{1}, x_{2}, x_{3}\right\}$ with, $\left(x_{1}, x_{2}, x_{3}\right)=$ three components color features of the chosen color space (i.e. Ohta, HSV, YCrCb etc). For each $x_{i}$, let $\mu\left(x_{i}\right)$ be the importance that takes the feature $x_{i}$ in the decision of the foreground detection process. The fuzzy functions $h\left(x_{i}\right)$ are defined in $[0,1]$ so that, $h\left(x_{1}\right)=S_{1}^{C}(x, y)$, $h\left(x_{2}\right)=S_{2}^{C}(x, y)$ and $h\left(x_{3}\right)=S_{3}^{C}(x, y)$. To simplify the computing, a lambda fuzzy measure is used to obtain the fuzzy measure of all subsets of criteria. To compute the value of the Choquet integral for each pixel, we need firstly to rearrange the features $x_{i}$ in the set $X$ with respect to the order: $h\left(x_{1}\right) \leq h\left(x_{2}\right) \leq h\left(x_{2}\right)$.

The pixel at position $(x, y)$ is considered as foreground if its Choquet integral value is less than a certain constant threshold $T h$ as follows:

$$
\text { if } C_{\mu}(x, y)<T h \text { then }(x, y) \text { is foreground }
$$

\section{ENFORCING SPATIAL CONSISTENCY}

Observations show that if a pixel is selected as foreground due to strong noise, it is unlikely that the neighboring pixels, both in time and space, are also affected by this noise. To address this issue, instead of threshold directly the Choquet integral at each pixel in the foreground detection, we compute the median of the Choquet integral of pixels in the 8-connected region surrounding the current pixel. Then the threshold is applied on the median of the Choquet integral, instead of the actual one. Finally, a connected component analysis is used to remove the remaining regions with a very small area.

\section{FUZZY ADAPTIVE BACKGROUND MAINTENANCE}

The background maintenance determines how the background will adapt itself to take into account the critical situations which can occurred. In the literature, there are two maintenance schemes: the blind one and the selective one.

\subsection{Blind and selective background maintenance}

The blind background maintenance consists to update all the pixels with the same rules which is usually an IIR filter as follows:

$$
B_{t+1}(x, y)=(1-\alpha) B_{t}(x, y)+\alpha I_{t}(x, y)
$$

where $\alpha$ is a leaning rate which is a constant in the interval $[0,1]$. The disadvantage of this scheme is that the value of pixels classified as foreground are taken into account in the computation of the new background and so polluted the background image. To solve this problem, some authors use a selective maintenance which consists of computing the new background image with a different learning rate following its previous classification into foreground or background as follows:

$$
\begin{aligned}
B_{t+1}(x, y)= & (1-\alpha) B_{t}(x, y)+\alpha I_{t}(x, y) \\
& \text { if }(x, y) \text { is background } \\
B_{t+1}(x, y)= & (1-\beta) B_{t}(x, y)+\beta I_{t}(x, y) \\
& \text { if }(x, y) \text { is foreground }
\end{aligned}
$$

Here, the idea is to adapt very quickly a pixel classified as background and very slowly a pixel classified as foreground. For this reason, $\beta<<\alpha$ and usually $\beta=0$. So the Equation (6) becomes:

$$
B_{t+1}(x, y)=B_{t}(x, y)
$$

But the problem is that erroneous classification results may make permanent incorrect background model. 


\subsection{Adaptive background maintenance}

The disadvantage of the selective maintenance is mainly due to the crisp decision which attributes a different rule following the classification in background or foreground. To solve this problem, we propose to take into account the uncertainty of the classification. This can be made by graduate the update rule using the result of the Choquet integral as follows:

$$
\begin{aligned}
B_{t+1}(x, y)= & \mu_{F} B_{t}+ \\
& \mu_{B}\left((1-\alpha) B_{t}(x, y)+\alpha I_{t}(x, y)\right)
\end{aligned}
$$

where $\mu_{F}=1-\mu_{B}$.

$\mu_{F}$ and $\mu_{B}$ are respectively the fuzzy membership values of the pixel $(x, y)$ to the class foreground and background. $\mu_{B}$ is a function of $C_{\mu}(x, y)$ such as $\mu_{B}=1$ for $\operatorname{Max}\left(C_{\mu}(x, y)\right)$ and $\mu_{B}=0$ for $\operatorname{Min}\left(C_{\mu}(x, y)\right)$.

We can remark that the adaptive maintenance is a generalized version of the selective maintenance. Indeed, if the pixel is classified as background with a Choquet integral value equal to one, we retrieve the Equation (5), and if the pixel is classified as foreground with a Choquet intergral value equal to zero, the Equation (8) is equal to the Equation (7). This fuzzy adaptive scheme is tested on the Wallflower test sequence in the section 5 .

\section{EXPERIMENTAL RESULTS}

We have applied our algorithm to different dasets: the first one is the PETS 2006 dataset ${ }^{1}$ used in video surveillance evaluation. The output images of this dataset are $720 \times 576$ pixels. The second one is the Wallflower dataset ${ }^{2}$, where the output images are $160 \times 120$. We show results of one video of each datasets due to limitation of page. We have tested different fuzzy density values and, by experimentation, best results are obtained with the measures: $(0.50,0.35,0.15)$.

\subsection{PETS 2006 dataset}

This dataset contains several video sequences of indoor sequences in video surveillance context. The goal is to detect moving persons or abandoned luggage. The Figure 1 shows the experiments made on one sequence. The Figure 1-(c) and 1-(d) show respectively the final foreground mask obtained by the crisp operator OR and the Choquet integral using the $\mathrm{YCrCb}$ color space. The results obtained using the Choquet integral give less false positive detection. Then, to see the effect of other color spaces, we have tested the algorithm based on Choquet integral with RGB and HSV color spaces (see figure 1-(e) and 1-(f)). Based on visual interpretation, the results obtained by the Choquet integral using $\mathrm{YCrCb}$ were better than the results obtained with RGB and HSV color spaces.

\footnotetext{
${ }^{1}$ http://www.cvg.rdg.ac.uk/PETS2006/data.html

${ }^{2}$ http://research.mocrosoft.com/jckrumm/wallflower/testimages.html
}

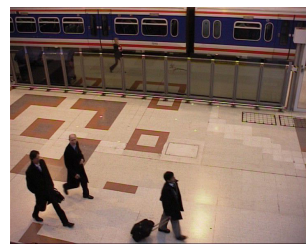

(a)

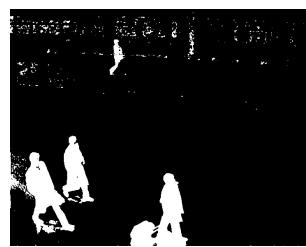

(c)

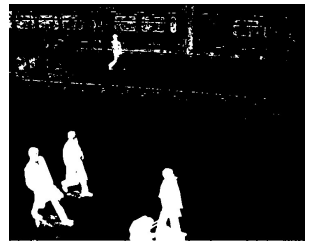

(e)

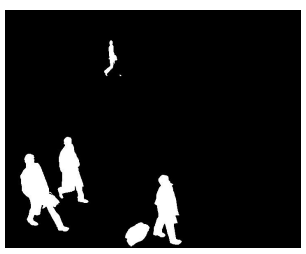

(b)

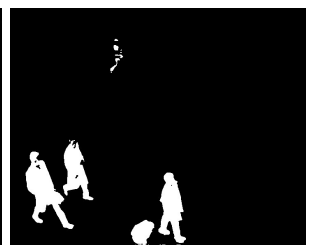

(d)

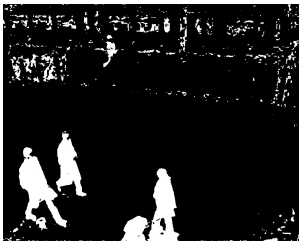

(f)
Fig. 1. (a) The current image, (b) the ground truth, (c) OR$\mathrm{YCrCb}$, (d) Choquet-YCrCb, (e) Choquet-RGB, (f) ChoquetHSV.

Table 1. Quantitative Evaluations

\begin{tabular}{|c|c|c|c|c|}
\hline $\begin{array}{c}\text { Operator } \\
\text { Color Space }\end{array}$ & $\begin{array}{c}\text { OR } \\
\text { YCrCb }\end{array}$ & $\begin{array}{c}\text { Choquet } \\
\text { YCrCb }\end{array}$ & $\begin{array}{c}\text { Choquet } \\
\text { RGB }\end{array}$ & $\begin{array}{c}\text { Choquet } \\
\text { HSV }\end{array}$ \\
\hline$S(A, B) \%$ & 68.14 & 77.89 & 65.35 & 54.91 \\
\hline
\end{tabular}

Numerical evaluation has been done quantitatively with respect to the similarity measure derived by Li et al. [16], noted $S(A, B)$, which compare the detected region $A$ and the corresponding region in the ground truth $B$. This quantity approaches 1 when $A$ and $B$ are similar and 0 otherwise. Table 1 shows the evaluation obtained for the previous experiments. It is well identified that optimum results are obtained with the fuzzy foreground detection using the $\mathrm{YCrCb}$ color space.

\subsection{Wallflower dataset}

This dataset contains 7 real video sequences presenting typical critical situations. Here, we show results on the sequence called Time of day, because it presents gradual illumination changes which alter the background and so permit to validate our fuzzy adaptive background maintenance. The Figure 2(c), 2-(d), and 2-(e) show respectively the final foreground mask obtained by the blind scheme the selective one and the proposed one. Table 2 shows the evaluation of the different update rules for the previous experiments. The fuzzy adaptive 


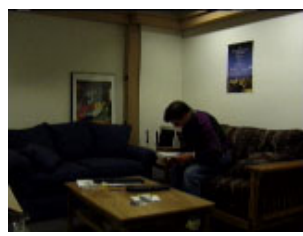

(a)

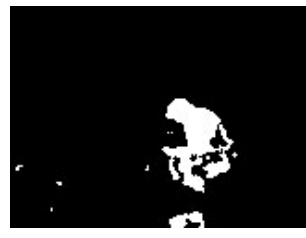

(c)

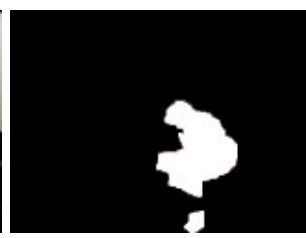

(b)

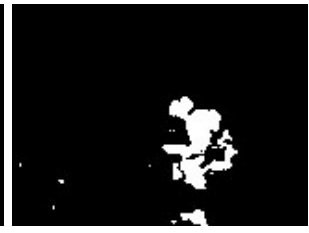

(d)

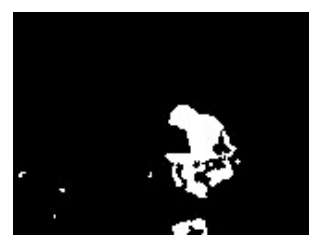

(e)

Fig. 2. (a) The current image, (b) the ground truth, (c) Blind Maintenance, (d) Selective Maintenance, (e) Adaptive Maintenance.

Table 2. Evaluation of the Maintenance scheme

\begin{tabular}{|c|c|c|c|}
\hline $\begin{array}{c}\text { Maintenance } \\
\text { scheme }\end{array}$ & $\begin{array}{c}\text { Blind } \\
\text { Maintenance }\end{array}$ & $\begin{array}{c}\text { Selective } \\
\text { Maintenance }\end{array}$ & $\begin{array}{c}\text { Adaptive } \\
\text { Maintenance }\end{array}$ \\
\hline$S(A, B) \%$ & 58.40 & 57.08 & 58.96 \\
\hline
\end{tabular}

scheme seems to be slightly better than the other update rules from the quantitative evaluation point of view, but it shows an improvement based on visual interpretation. The other maintenance rules are particular cases of the proposed scheme.

\section{CONCLUSION}

In this paper, we have presented a foreground detection method using the Choquet integral for fusing color features. Then, we have proposed a fuzzy adaptive maintenance rule to take into account the uncertainty of the classification. Experiments on video surveillance datasets show that this approach is robust and simple to implement. Further research consists in fusing other features and learning the fuzzy measures.

\section{REFERENCES}

[1] H. Zhang, D. Xu, "Fusing Color and Texture Features for Background Model," Third ICFSKD 2006, vol. 4223, no. 7, pp. 887-893, Sept. 2006.
[2] F.El Baf, T. Bouwmans, "Comparison of Background Subtraction Methods for a Multimedia Learning Space," ICSPM 2007, Jul. 2007.

[3] S. Cheung, C. Kamath, "Robust Background Subtraction With Foreground Validation for Urban Traffic Video," EURASIP Journal on Applied Signal Processing, Sept. 2005.

[4] J. Carranza , M. MAgnor, "Free-Viewpoint Video of Human Actors," ACM Transactions on Graphics, vol. 22, no. 3, pp. 569-577, 2003.

[5] B. Lee, M. Hedley "Background Estimation for Video Surveillance," IVCNZ 2002, pp. 315-320, 2002.

[6] N. McFarlane, C. Schofield, "Segmentation and tracking of piglets in images," BMVA 1995, pp. 187-193, 1995.

[7] J. Zheng, Y. Wang, "Extracting Roadway Background Image: A mode based approach," Transportation Research Board, 2006.

[8] C Wren, A. Azarbayejani, "Pfinder : Real-Time Tracking of the Human Body," IEEE PAMI 1997, vol. 19, no. 7, pp. 780 -785, Jul. 1997.

[9] C. Stauffer, "Adaptive background mixture models for real-time tracking," IEEE CVPR 1999, pp. 246-252, 1999.

[10] A. Elgammal, L. Davis, "Non-parametric Model for Background Subtraction," 6th ECCV 2000, Jun. 2000.

[11] K. Toyama, J. Krumm, "Wallflower: Principles and Practice of Background Maintenance," ICCV 1999, Sept. 1999.

[12] S. Messelodi, C. Modena, "A Kalman filter based background updating algorithm robust to sharp illumination changes," ICIAP 2005, Sept. 2005.

[13] R. Chang, T. Ghandi, M. Trivedi, "Vision modules for a multi sensory bridge monitoring approach," IEEE CITS 2004 , Oct 2004.

[14] Y. Narukawa, T. Murofushi, "Decision Modelling Using the Choquet Integral," MDAI 2004, vol. 3131, pp. 183193, 2004.

[15] M. Sugeno, S. Kwon, "A new approach to time series modeling with fuzzy measures and the Choquet integral," 4th IEEE ICFS 1995, pp. 799-804, Mar. 1995.

[16] L. Li, W. Huang, "Statistical Modeling of Complex Background for Foreground Object Detection," IEEE TIP 2004, vol. 13, no. 11 pp. 81459-1472, Nov. 2004.

[17] F.El Baf, T. Bouwmans, B. Vachon "Foreground Detection using the Choquet Integral," WIAMIS 2008, May 2008. 